\section{Acesso de pessoas deficientes auditivas a serviços de saúde em cidade do Sul do Brasil}

\author{
Access by hearing-disabled individuals to health \\ services in a southern Brazilian city
}

\author{
${ }_{1}$ Universidade Luterana do \\ Brasil, Canoas, Brasil. \\ 2 Universidade Federal da \\ Bahia, Salvador, Brasil. \\ Correspondência \\ D. B. Freire \\ Programa de Pós-gradu- \\ ação em Saúde Coletiva, \\ Universidade Luterana do \\ Brasil. \\ Av. Farroupilha 8001 \\ Canoas, $R S$ \\ 92425-900, Brasil. \\ daniela.buchrieser@gmail.com
}

\begin{abstract}
This cross-sectional study aimed to compare access to health services and preventive measures by persons with hearing disability and those with normal hearing in Canoas, Rio Grande do Sul State, Brazil. The sample included 1,842 individuals 15 years or older (52.9\% of whom were females). The most frequent income bracket was twice the minimum wage or more, or approximately U\$360/month (42.7\%). Individuals with hearing disability were more likely to have visited a physician in the previous two months $(P R=$ 1.3, 95\%CI: 1.10-1.51) and to have been hospitalized in the previous 12 months ( $P R=2.1,95 \%$ CI: 1.42-3.14). Regarding mental health, individuals with hearing disability showed 1.5 times greater probability of health care due to mental disorders and 4.2 times greater probability of psychiatric hospitalization as compared to those with normal hearing. Consistent with other studies, women with hearing disability performed less breast self-examination and had fewer Pap smears. The data indicate the need to invest in specific campaigns for this group of individuals with special needs.
\end{abstract}

Hearing Loss; Health Services Accessibility; Sexual Behavior

\author{
Daniela Buchrieser Freire 1 \\ Luciana Petrucci Gigante 1 \\ Jorge Umberto Béria 1 \\ Lílian dos Santos Palazzo 1 \\ Andréia Cristina Leal Figueiredo 1,2 \\ Beatriz Carmen Warth Raymann 1
}

\section{Introdução}

A Organização Mundial da Saúde (OMS) estimou que, em 2005, havia 278 milhões de pessoas no mundo com deficiência auditiva moderada a profunda. A maioria vive em países subdesenvolvidos ou em desenvolvimento, e o número de indivíduos com essa deficiência vem crescendo em todo o mundo principalmente pelo aumento da população mundial e da expectativa de vida ${ }^{1}$. Os dados do Censo Demográfico de 2000 descrevem uma prevalência de 3,5\% de algum tipo de deficiência auditiva na população brasileira 2. No Reino Unido, o envelhecimento da população acarretará um aumento aproximado de $20 \%$ de deficientes auditivos nos próximos vinte anos. A perda auditiva é freqüentemente subdiagnosticada, especialmente nos idosos 3 .

Como qualquer outro grupo populacional, as pessoas com deficiência auditiva precisam de acesso à saúde, não necessariamente relacionado com a deficiência em si ${ }^{4}$. A orientação para medidas de prevenção pode ser feita em nível populacional, na forma de políticas ou campanhas de divulgação na mídia, ou em nível individual, nas consultas com profissionais da saúde. As orientações devem estar de acordo com as características socioculturais da população-alvo, e estas devem ser respeitadas para que se consiga atingir o objetivo ${ }^{5}$. A comunicação com esses pacientes é um desafio para os profissionais da saúde 6 , já que o atendimento adequado 
de pessoas com necessidades especiais é essencial para a qualidade dos serviços de saúde, enquanto a falta de comunicação impede um atendimento humanizado.

As informações sobre saúde são adquiridas em diferentes meios, como família, amigos, rádio, televisão, materiais escritos e na Internet. Indivíduos que se tornaram deficientes auditivos antes de adquirir a fala (pessoas com surdez prélingual) têm maior dificuldade para adquirir a linguagem, ler e escrever, o que limita sua aquisição de informações em várias fontes 7 . Além disso, a maioria das pessoas com deficiência auditiva está nas classes menos favorecidas, o que também limita o acesso à informação de alguma forma.

A comunicação com deficientes auditivos é um grande obstáculo para os profissionais da saúde, prejudicando vínculo e atendimento desses pacientes 3,6 . Reconhecer a deficiência na comunicação em situação de cuidado à saúde leva os profissionais a refletirem sobre essas questões e a melhorarem os serviços 4,8 .

O presente artigo tem como objetivo comparar o acesso dos portadores de deficiência auditiva incapacitante a serviços assistenciais e de prevenção com o acesso dos ouvintes.

\section{Método}

Utiliza-se de um banco de dados resultante de uma pesquisa de base populacional realizada na cidade de Canoas, Rio Grande do Sul, Brasil, que abordou diversos aspectos da saúde da população. Como parte do estudo, mediu-se a audição de todos os participantes da investigação, da seguinte forma: audiometria, para pessoas com quatro anos ou mais; observação comportamental, para menores de quatro anos; emissões otoacústicas, para menores de seis meses.

$\mathrm{O}$ estudo, transversal, foi realizado em Canoas, cidade metropolitana de Porto Alegre. Os dados foram colhidos de novembro de 2002 a julho de 2003. Nesse período, a população da cidade era de 306.093 habitantes, moradores em domicílios particulares permanentes, distribuídos pelo Instituto Brasileiro de Geografia e Estatística (IBGE) em 391 setores censitários.

A amostra foi calculada para um estudo transversal de base populacional com prevalência do desfecho deficiência auditiva incapacitante de $10 \%$, nível de $95 \%$ de confiança e efeito de delineamento 2. Isso resultou em uma amostra de 1.040 domicílios (esperando 3,75 pessoas por domicílio, segundo dados do IBGE; http:// www.ibge.gov.br, acessado em Out/2007).
Para contemplar toda a cidade, foram sorteados 40 dos 391 setores censitários do Município de Canoas. Em cada setor foi sorteado um quarteirão e, em seguida, uma esquina deste. A partir da esquina sorteada, 26 domicílios foram visitados (um a cada quatro), nos quais todos os moradores eram convidados a participar.

Foram excluídos da pesquisa os que não quiseram participar e os que estavam ausentes em três visitas realizadas em dias e horários diferentes. Outros participantes foram excluídos por motivo de doença que os incapacitava de responder ao questionário e/ou realizar o exame audiométrico.

$\mathrm{O}$ instrumento da pesquisa era um questionário pré-codificado, padronizado e previamente testado, aplicado aos moradores de 14 anos ou mais. O questionário incluía um instrumento auto-aplicável e confidencial, a respeito de aspectos de comportamento sexual. Utilizou-se o protocolo de pesquisa de distúrbios de orelha e audição da OMS (http://www.who.int, acessado em Out/2007), para pesquisas de base populacional de prevalência e causas de surdez e deficiência auditiva e outras doenças da orelha.

No estudo-piloto, um bolsista estudante de medicina aplicou o questionário a cinqüenta pessoas de diversas idades, em Porto Alegre, para avaliar a linguagem e a lógica. A partir desse teste, foram realizadas algumas adequações na linguagem.

O controle de qualidade foi realizado em $11,6 \%$ dos domicílios, os quais receberam ou uma revisita ou um telefonema, para confirmar se tinham sido submetidos à audiometria e responder a mais duas perguntas que compunham o questionário. A confiabilidade entre os resultados do controle de qualidade e as entrevistas originais foi medida pelo kappa que foi de 0,93.

Todos os participantes assinaram termo de consentimento livre e esclarecido. Os nomes e os dados dos participantes foram mantidos em sigilo. O projeto foi submetido ao Comitê de Ética em pesquisa em seres humanos e animais da Universidade Luterana do Brasil, em abril de 2002, sendo considerado aprovado sob o número 049-2002. Os participantes com exames alterados foram orientados pelos pesquisadores a buscar serviços de saúde para atendimento médico especializado.

As informações foram digitadas em um programa que foi desenvolvido para garantir que os passos lógicos do questionário fossem realizados e impedir que dados fora da amplitude prevista fossem digitados. Foi realizada dupla digitação independente para verificar inconsistências. O programa gerou um relatório de erros, com base no qual as informações foram 
corrigidas, conforme os questionários, antes da análise.

A população-alvo do presente estudo são os indivíduos com 15 anos ou mais. A amostra estudada incluiu um total de 1.842 pessoas nessa faixa etária, que foram entrevistadas para o primeiro estudo.

As variáveis foram divididas em categorias: sócio-demográficas (idade, sexo, viver com esposo/a ou companheiro/a, renda no mês anterior à entrevista, saber ler e escrever, anos de escolaridade), relativas a serviços de saúde (ter convênio ou plano de saúde, consulta médica nos últimos dois meses, internação hospitalar no último ano), relativas a medidas de prevenção (tempo decorrido desde o último citopatológico de colo de útero, tempo decorrido desde o último exame de próstata, uso de camisinha na última relação sexual), relativas a aspectos de saúde (hábito de realizar auto-exame de mamas, realização de consulta por problema de nervos, internação em hospital psiquiátrico, avaliação da chance de pegar AIDS), eventos estressantes (ter perdido emprego no último ano, ter se separado do companheiro no último ano, ter sido assaltado ou roubado no último ano). Foi avaliado o efeito de ser portador de deficiência auditiva incapacitante sobre cada uma das variáveis acima.

Segundo a OMS (http://www.who.int, acessado em Out/2007), são consideradas perdas auditivas incapacitantes aquelas que são permanentes e em que, sem amplificação, a média da percepção auditiva entre as freqüências 0,5, 1, 2 e $4 \mathrm{kHz}$ se situa acima de $31 \mathrm{~dB}$ para crianças até 15 anos e $41 \mathrm{~dB}$ para pessoas acima de 15 anos e adultos na melhor orelha.

Os dados foram analisados no programa SPSS (SPSS Inc., Chicago, Estados Unidos). A análise estatística foi feita em etapas: análise descritiva e análise bivariada. Para tentar minimizar o efeito das perdas, que foram maiores no grupo de homens de 20 a 39 anos de idade, utilizou-se a ponderação por sexo e idade nas análises ${ }^{9}$. Para a análise descritiva, foi verificada a freqüência das variáveis qualitativas e a média e desvio-padrão (DP) para as variáveis quantitativas. A segunda etapa da análise comparou as freqüências das variáveis estudadas entre os portadores de deficiência auditiva incapacitante e os ouvintes, utilizando-se, para isso, o qui-quadrado. Para as variáveis quantitativas, foi usado teste T de Student, comparando as médias nos diferentes grupos de capacidade auditiva. O nível de significância escolhido foi de $5 \%$. Foram calculadas também as razões de prevalência e seus intervalos de $95 \%$ de confiança (IC95\%).

\section{Resultados}

A amostra incluiu 1.842 pessoas com 15 anos ou mais, sendo a maioria mulheres (52,9\%). A Tabela 1 mostra a distribuição da amostra conforme as variáveis sócio-demográficas estudadas. Chama a atenção a escolaridade de 11 anos ou mais $(33,7 \%)$ e $96,4 \%$ das pessoas alfabetizadas. A renda individual de dois salários mínimos ou mais foi a mais freqüente $(42,7 \%)$. Mais da metade dos entrevistados (62,2\%) vive com companheiro/a.

Foi avaliado o efeito de ser portador de deficiência auditiva incapacitante sobre cada uma das variáveis qualitativas descritas na Tabela 2.

Quando analisadas as variáveis relativas a serviços de saúde, os portadores de deficiência auditiva apresentaram $30 \%$ mais prevalência de consulta nos dois meses anteriores à entrevista do que os ouvintes (RP: 1,3; IC95\%: 1,10-1,51).

Quanto ao local da última consulta, os deficientes auditivos tiveram maior probabilidade de consultar em hospital ou em outra cidade do que os ouvintes (RP = 1,5; IC95\%: 1,1-2,1). Tiveram 2,1

Tabela 1

Distribuição da amostra segundo características sócio-demográficas. Canoas, Rio Grande do Sul, Brasil, 2003 *

\begin{tabular}{|c|c|c|}
\hline Característica & $\mathbf{n}$ & $\%$ \\
\hline \multicolumn{3}{|l|}{ Sexo } \\
\hline Masculino & 868 & 47,1 \\
\hline Feminino & 974 & 52,9 \\
\hline \multicolumn{3}{|c|}{ Faixas etárias (anos) } \\
\hline $15-19$ & 221 & 12,0 \\
\hline $20-39$ & 834 & 45,3 \\
\hline $40-59$ & 569 & 30,9 \\
\hline 60 ou mais & 218 & 11,8 \\
\hline \multicolumn{3}{|c|}{ Viver com esposo/a ou companheiro/a } \\
\hline Sim & 1.123 & 62,2 \\
\hline Não & 684 & 37,8 \\
\hline \multicolumn{3}{|c|}{ Renda no mês anterior à entrevista (salários mínimos) } \\
\hline Menos de 1 & 587 & 32,0 \\
\hline $1-2$ & 464 & 25,3 \\
\hline 2 ou mais & 783 & 42,7 \\
\hline \multicolumn{3}{|l|}{ Alfabetização } \\
\hline Sim & 1.775 & 96,4 \\
\hline Não & 66 & 3,6 \\
\hline \multicolumn{3}{|c|}{ Anos de escolaridade (anos completos) } \\
\hline $0-3$ & 308 & 16,7 \\
\hline $4-7$ & 546 & 29,7 \\
\hline $8-10$ & 367 & 19,9 \\
\hline 11 ou mais & 621 & 33,7 \\
\hline
\end{tabular}

* Dados ponderados. 
Associação entre características qualitativas estudadas e a deficiência auditiva. Canoas, Rio Grande do Sul, Brasil, 2003 *

\begin{tabular}{|c|c|c|c|c|c|c|c|}
\hline \multirow[t]{2}{*}{ Características } & \multicolumn{2}{|c|}{$\begin{array}{l}\text { Pessoas com deficiência } \\
\text { auditiva incapacitante }\end{array}$} & \multicolumn{2}{|c|}{ Ouvintes } & \multirow[t]{2}{*}{$\begin{array}{l}\text { Razão de } \\
\text { prevalências }\end{array}$} & \multirow[t]{2}{*}{ IC95\% } & \multirow[t]{2}{*}{$\begin{array}{l}\text { Valor } \\
\text { de } p\end{array}$} \\
\hline & $\mathrm{n}$ & $\%$ & $\mathrm{n}$ & $\%$ & & & \\
\hline \multicolumn{8}{|l|}{ Relativas a serviços de saúde } \\
\hline Possuir convênio ou plano de saúde & & & & & & & 0,106 \\
\hline Sim & 89 & 64,0 & 962 & 56,6 & 1,1 & $0,99-1,29$ & \\
\hline Não & 50 & 36,0 & 738 & 43,4 & 1,0 & & \\
\hline Consulta nos últimos 2 meses & & & & & & & 0,007 \\
\hline Sim & 77 & 55,4 & 731 & 43,1 & 1,3 & $1,10-1,51$ & \\
\hline Não & 62 & 44,6 & 965 & 56,9 & 1,0 & & \\
\hline Local da última consulta & & & & & & & 0,038 \\
\hline Ambulatório em Canoas & 51 & 66,2 & 562 & 77,5 & 0,85 & $0,72-1,0$ & \\
\hline Hospital ou outra cidade & 26 & 33,8 & 163 & 22,5 & 1,0 & & \\
\hline Internação hospitalar no último ano & & & & & & & 0,001 \\
\hline Sim & 24 & 17,4 & 140 & 8,2 & 2,1 & $1,42-3,14$ & \\
\hline Não & 114 & 82,6 & 1.561 & 91,8 & 1,0 & & \\
\hline \multicolumn{8}{|l|}{ Relativas a medidas de prevenção } \\
\hline Uso de preservativo na última relação sexual & & & & & & & 0,002 \\
\hline Sim & 25 & 22,3 & 563 & 37,1 & 0,6 & $0,42-0,86$ & \\
\hline Não & 87 & 77,7 & 954 & 62,9 & 1,0 & & \\
\hline Hábito de realizar auto-exame de mamas & & & & & & & 0,020 \\
\hline Sim & 37 & 56,1 & 632 & 70,5 & 0,8 & $0,64-0,99$ & \\
\hline Não & 29 & 43,9 & 264 & 29,5 & 1,0 & & \\
\hline \multicolumn{8}{|l|}{ Relativas à saúde mental } \\
\hline Consulta por problemas de nervos & & & & & & & 0,003 \\
\hline Sim & 47 & 33,8 & 378 & 22,2 & 1,5 & $1,18-1,95$ & \\
\hline Não & 92 & 66,2 & 1.323 & 77,8 & 1,0 & & \\
\hline Internação em hospital psiquiátrico & & & & & & & 0,000 \\
\hline Sim & 11 & 7,9 & 32 & 1,9 & 4,2 & $2,17-8,16$ & \\
\hline Não & 128 & 92,1 & 1.668 & 98,1 & 1,0 & & \\
\hline Eventos estressantes ** & & & & & & & 0,089 \\
\hline Sim & 26 & 19,0 & 440 & 25,9 & 0,7 & $0,51-1,04$ & \\
\hline Não & 111 & 81,0 & 1.256 & 74,1 & 1,0 & & \\
\hline
\end{tabular}

* Dados ponderados.

** Assalto, separação e perda de emprego no ano anterior a entrevista.

vezes (IC95\%: 1,42-3,14) mais prevalência de internação hospitalar no ano anterior à entrevista do que os ouvintes.

No que diz respeito a medidas de prevenção, os deficientes auditivos tiveram $40 \%(\mathrm{RP}=0,6$; IC95\%: 0,42-0,86) menos prevalência de uso de preservativo na última relação sexual do que os ouvintes. As mulheres com deficiência também tiveram 20\% (RP = 0,8; IC95\%: 0,64-0,99) menos prevalência de realização de auto-exame das mamas do que as ouvintes.

Nas variáveis relativas a aspectos de saúde mental, aqueles com deficiência auditiva tiveram 4,2 (IC95\%: 2,17-8,16) vezes mais prevalência de internação por problema de nervos que os ouvintes. Por outro lado, não houve diferença estatística quanto a eventos estressantes no último ano na comparação dos dois grupos $(\mathrm{RP}=$ 0,7; IC95\%: 0,51-1,04).

As variáveis quantitativas estão descritas na Tabela 3. A média de anos decorridos desde o último citopatológico de colo uterino realizado pelas mulheres deficientes auditivas (média: 24,3 anos; DP = 32,99) foi muito superior à média encontrada para as ouvintes (média 7,2 anos, $\mathrm{DP}=13,81 ; \mathrm{p}=0,000$ ). Porém, em relação aos homens com mais de 40 anos, o tempo decorrido desde o último exame de próstata não apresentou diferença entre os grupos. 
Quando questionados sobre sua chance de pegar AIDS, os deficientes auditivos responderam em sua maioria ser impossível pegar AIDS $(60,6 \%)$, respondendo apenas $5,1 \%$ ser possível, como pode ser observado na Figura 1.

Quando os dados são estratificados por faixas etárias (15 a 19 anos, 20 a 39 anos, 40 a 59 anos, 60 anos ou mais), a situação se modifica, sendo a única faixa que apresenta diferença estatisticamente significante, a faixa de 20 a 39 anos. A análise de resíduos mostra que há diferença entre os grupos, os deficientes acham que é muito possível pegar AIDS (14,3\%) comparado com os ouvintes 3,3\% (Figura 2).

\section{Discussão}

O presente estudo tem delineamento transversal, logo os resultados obtidos devem ser vistos como fatores associados e não como fatores de risco. Este tipo de estudo está sujeito à causalidade reversa, não sendo possível avaliar a temporalidade entre causa e efeito 10 .

Como o estudo apresentou uma considerável proporção de perdas (20,3\%), que se concentrou mais no grupo de homens de 20 a 39 anos de idade, foi utilizado um fator de correção na análise dos dados, ponderando-se os resultados por sexo e idade ${ }^{9}$.

Tabela 3

Avaliação da diferença entre as médias das variáveis quantitativas relacionadas aos deficientes auditivos e aos indivíduos sem deficiência auditiva. Canoas, Rio Grande do Sul, Brasil, 2003 *

\begin{tabular}{|c|c|c|c|c|c|}
\hline \multirow[t]{2}{*}{ Variáveis } & \multicolumn{2}{|c|}{$\begin{array}{l}\text { Pessoas com deficiência } \\
\text { (homens, } \mathrm{n}=60 ; \text { mulheres, } \mathrm{n}=66 \text { ) }\end{array}$} & \multicolumn{2}{|c|}{$\begin{array}{l}\text { Ouvintes } \\
\text { (homens, } \mathrm{n}=291 ; \text { mulheres, } \mathrm{n}=\mathbf{8 9 3} \text { ) }\end{array}$} & \multirow[t]{2}{*}{$\begin{array}{l}\text { Valor } \\
\text { de } p\end{array}$} \\
\hline & Média (em anos) & DP & Média (em anos) & DP & \\
\hline \multicolumn{6}{|l|}{ Relativas a medidas de prevenção } \\
\hline Tempo decorrido desde o último citopatológico & 24,3 & 32,99 & 7,2 & 13,81 & 0,000 \\
\hline Tempo decorrido desde último exame de próstata & 30,6 & 28,62 & 30,5 & 24,14 & 0,980 \\
\hline
\end{tabular}

* Dados ponderados.

Figura 1

Percepção da chance de pegar AIDS. Canoas, Rio Grande do Sul, Brasil, 2003.

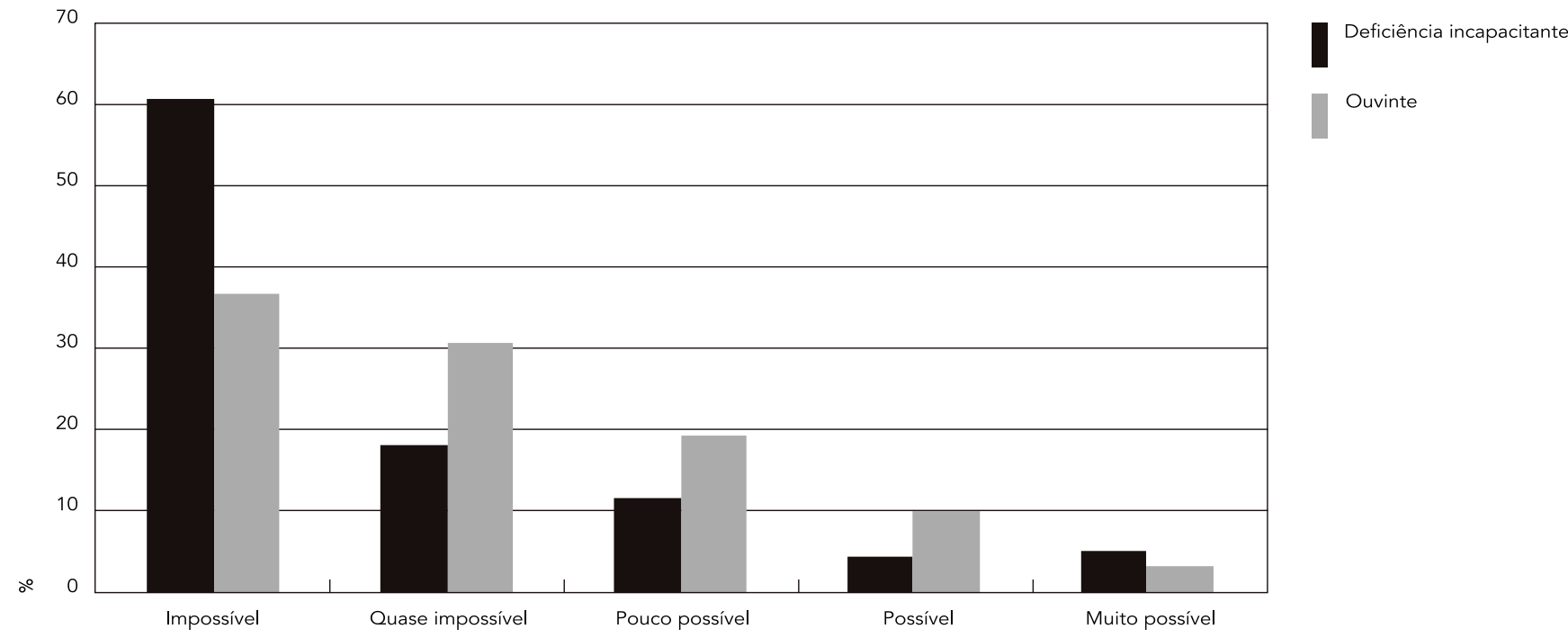


Figura 2

Percepção da chance de pegar AIDS na faixa etária de 20 a 39 anos. Canoas, Rio Grande do Sul, Brasil, 2003.

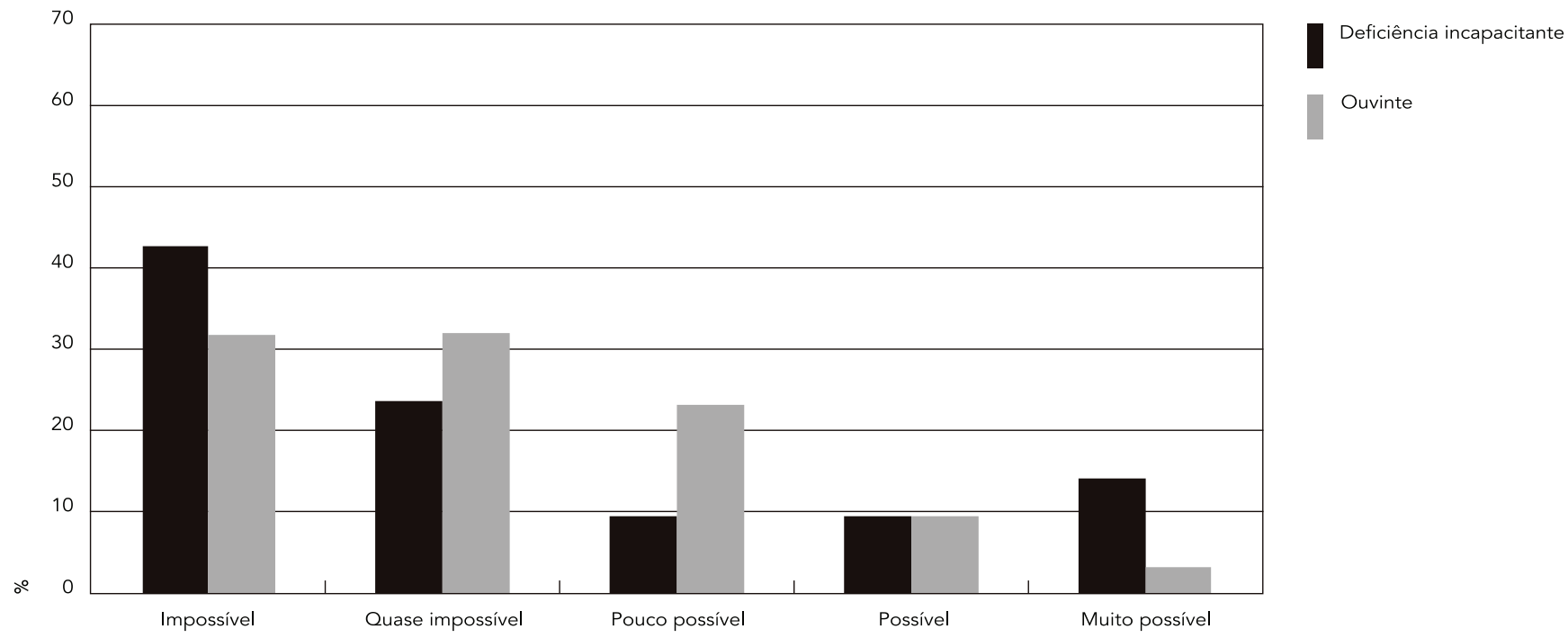

Nas variáveis sócio-demográficas, os entrevistados mostraram que eram, em sua maioria, alfabetizados $(96,4 \%)$, com 11 anos ou mais de estudo (33,7\%). A taxa de analfabetismo é semelhante à da Região Sul $(7,8 \%)$ e inferior à taxa brasileira (13,3\%). Em relação a anos de estudo, a taxa encontrada (11 anos ou mais; 33,7\%) foi muito superior à da Região Sul (6,2 anos) e do Brasil (5,7 anos). A renda individual mais freqüente de dois salários mínimos ou mais $(42,7 \%)$ corresponde à mais freqüente na Região Sul e no Brasil (IBGE. http://www.ibge.gov.br, acessado em Out/2007). Mais da metade dos entrevistados (62,2\%) vivia com companheiro/a.

Pessoas com deficiência auditiva poderão apresentar dificuldades na aquisição da linguagem, na fala e, por vezes, poderão apresentar também dificuldades nas habilidades cognitivas, levando ao isolamento. Indivíduos surdos têm condições de saúde inferiores em relação a ouvintes e parecem acessar os serviços de saúde de maneira diferente 11. Por essas razões, também podem acessar menos e usar inadequadamente medidas de prevenção, como o exame preventivo de colo de útero e a mamografia ${ }^{12}$. Quando analisadas as variáveis relativas a serviços de saúde nos portadores de deficiência auditiva incapacitante neste estudo, a prevalência de ter convênio ou plano de saúde foi maior do que entre os ouvintes. O mesmo se deu com relação a con- sultas nos dois meses anteriores à entrevista: os deficientes apresentaram $30 \%$ mais prevalência do que os ouvintes. Esse achado mostra a maneira diferente de acessar os serviços, com os deficientes auditivos consultando mais talvez por não terem seus problemas resolvidos da maneira adequada.

Quanto ao local da última consulta, os portadores de deficiência auditiva tiveram maior prevalência de consulta em hospital ou em outra cidade do que os ouvintes; em adição, aqueles tiveram 2,1 vezes mais prevalência de internação hospitalar no ano anterior à entrevista do que estes.

Esses resultados podem refletir a dificuldade de acesso a serviços ambulatoriais de maneira geral, levando as pessoas a procurarem o nível terciário; podem revelar também a dificuldade de se atingirem essas pessoas com medidas preventivas.

Cardoso et al. 4, em um estudo de análise qualitativa, descrevem que as razões mais freqüentes de busca aos serviços de saúde, por pessoas com surdez severa ou profunda, foram: dor de cabeça, dor de estômago, motivos relacionados à gestação, dor de ouvido e tosse. Todas estas são queixas que poderiam ser manejadas no nível ambulatorial. No presente estudo, não foi possível identificar os motivos das consultas, mas é provável que sejam os mesmos que levam deficien- 
tes auditivos e ouvintes a procurarem o médico, excetuando razões referentes à própria deficiência. Diante disso, não haveria justificativa médica para os deficientes auditivos procurarem mais serviços afastados de sua residência ou de nível terciário a não ser o fato de que provavelmente eles estão em contato com esses serviços em razão de sua deficiência. Portanto, não há um bom funcionamento do sistema de contra-referência, ou a assistência no nível primário não está conseguindo satisfazer suas necessidades.

Um trabalho realizado com mulheres surdas em Rochester, Estados Unidos, que tinham como primeira língua a Língua Americana de Sinais e eram líderes em suas comunidades, mostrou que a maioria desconhecia o significado ou a importância de medidas de prevenção, rastreamento de neoplasias, mamografia, exame preventivo do colo de útero, terapia de reposição hormonal ou auto-exame das mamas. A maioria acredita que tem menor acesso a informações em saúde que as ouvintes e afirma que se mantém informada principalmente com familiares e amigos 12. Outro estudo com mulheres de diferentes tipos de deficiência auditiva confirma esses achados. Também confirma o fato de as pacientes sentirem-se envergonhadas, com medo de consultas e procedimentos em saúde, desconhecendo termos médicos e seus próprios corpos 3 . O mesmo artigo nota ainda que a maioria dos serviços de saúde, inclusive clínicas especializadas em audiologia, trabalha com um sistema de chamar em voz alta os nomes dos pacientes, o que limita o acesso destes e gera ansiedades e preocupações 3,6,8.

No presente estudo, no que diz respeito a medidas de prevenção, os deficientes auditivos tiveram $40 \%$ menos prevalência de usar preservativo na última relação sexual do que os ouvintes. As mulheres com deficiência tiveram $20 \%$ menos prevalência de realizar auto-exame das mamas do que as não deficientes, e a média de anos decorridos desde o último citopatológico de colo uterino nas mulheres deficientes foi muito superior à das ouvintes. Tais achados confirmam os dados da literatura, entretanto, em relação aos homens, o tempo decorrido desde o último exame de próstata não apresentou diferença entre os grupos. Como os homens que responderam a esta pergunta tinham mais de quarenta anos e, neste estudo, não sabemos a idade de início da perda auditiva, talvez esses homens já houvessem adquirido a informação da importância deste exame para sua saúde.

Trabalhos citam a associação de deficiência auditiva com depressão e outros transtornos mentais. Apesar de parecerem estar mais propensos a patologias mentais, os deficientes auditivos têm menos acesso aos cuidados especializados $3,13,14$. Na pesquisa de Steinberg et al. 15 com portadores de deficiência auditiva incapacitante, poucos atribuíram a deficiência a problemas mentais, porém quase a metade associou a dificuldade de comunicação, problemas familiares e o preconceito social que acompanha esse tipo de deficiência a problemas como depressão, abuso de substâncias e comportamento violento.

No estudo aqui apresentado, os deficientes auditivos tiveram 1,5 vez mais prevalência de ter consultado por problema de nervos e 4,2 vezes mais prevalência de terem sido internados por problema de nervos do que os ouvintes. Jones et al. 16 mostraram que aqueles têm maiores níveis de estresse percebido do que a população geral e que o manejo adequado pode diminuir esse nível de estresse e doenças relacionadas a ele. Não se evidenciou, também, diferença estatística quanto a eventos estressantes no último ano na comparação dos dois grupos. Os eventos estudados foram assalto, separação e perda de emprego. Não foram investigados os problemas familiares, nem o abuso de substâncias, mas a maioria das pessoas tinha companheiro/a, além de bom nível econômico e educacional, o que pode protegê-los do estresse.

Considerando que pessoas portadoras de deficiência auditiva incapacitante apresentam dificuldade no acesso a informações em saúde, a questão se torna ainda mais delicada quando o assunto é sexualidade. Job 17 revisou literatura sobre surdez e sexualidade e verificou que o conhecimento da população surda estava muito inadequado. Associou esse achado a vários fatores, como falta de comunicação entre pais e filhos e na escola. O mesmo artigo cita uma pesquisa feita por Joseph 18 em 1995, que mostrou que estudantes, quando perguntados sobre seu entendimento da sexualidade humana, responderam corretamente à metade das perguntas a respeito de ovulação, HIV, reprodução e anticoncepção. Em outro estudo, realizado por Luckner \& Gonzáles 19, em 1993, com adolescentes surdos, encontraram-se os seguintes resultados: $70 \%$ acreditavam poder pegar AIDS doando sangue, $46 \%$ responderam que pessoas não heterossexuais não poderiam pegar AIDS e 43\% disseram que todos os homossexuais têm AIDS. Ainda $62 \%$ dos entrevistados acreditam que pessoas casadas não podem pegar AIDS 17. Neste trabalho, os resultados confirmam a literatura, mostrando que os deficientes auditivos utilizaram menos preservativos nas relações sexuais e, quando perguntados sobre qual sua chance de pegar AIDS, a maioria considera impossível (60,6\%). Já na estratificação por idades, na faixa 
etária de 20 a 39 anos, a situação se modifica e os deficientes responderam ser muito possível pegar AIDS. Isso pode refletir alguma mudança no acesso a informação e cuidado com a saúde da população deficiente, indicando que este pode ser o caminho para uma mudança na realidade de informações precárias e exposição a risco.

Os achados do presente estudo e a bibliografia revisada reforçam a necessidade de investimentos em campanhas específicas para deficientes auditivos para que estes tenham o mesmo acesso aos serviços de saúde e medidas de prevenção que os ouvintes, o que já lhes é garantido por lei 20,21 . Os serviços de saúde, se não puderem

\section{Resumo}

Este estudo transversal, de base populacional, tem como objetivo comparar o acesso dos portadores de deficiência auditiva incapacitante a serviços assistenciais e de prevenção com os ouvintes de Canoas, Rio Grande do Sul, Brasil. A amostra incluiu 1.842 pessoas com 15 anos ou mais, sendo a maioria mulheres (52,9\%). A renda individual de dois salários mínimos ou mais foi a mais freqüente (42,7\%). As pessoas com deficiência auditiva incapacitante tiveram $30 \%$ maior probabilidade de ter consultado nos dois meses anteriores à entrevista $(R P=1,3$, IC95\%: 1,10-1,51) e ter sido internado nos 12 meses anteriores à entrevista $(R P=$ 2,1, IC95\%: 1,42-3,14). Nas variáveis relativas à saúde mental, os deficientes auditivos tiveram 1,5 mais probabilidade de terem consultado para problemas de nervos (IC95\%: 1,18-1,95) e 4,2 mais probabilidades de terem sido internados em hospital psiquiátrico que os ouvintes (IC95\%: 2,17-8,16). Assim como encontrado na literatura, as mulheres deficientes auditivas realizaram menos auto-exame das mamas e exame citopatológico de colo uterino. Os dados encontrados indicam a necessidade de investir em educação em saúde e campanhas específicas para o grupo de pessoas investigado.

Perda Auditiva; Acesso aos Serviços de Saúde; Comportamento Sexual dispor de profissionais habilitados a se comunicar em Língua Brasileira de Sinais (LIBRAS) ou dispor de intérpretes, devem, pelo menos, ter o cuidado de atender a essas pessoas da melhor forma possível, para que tenham suas necessidades atendidas de maneira integral. Apesar de já existir legislação que prevê esta atenção, os próprios deficientes se colocam em uma posição de diferença, mostrando atitude de "conformismo $e$ culpabilização das próprias pessoas surdas pelas limitações vivenciadas", como descreve Marin \& Góes 22 (p. 244). Cabe a toda a sociedade, em especial aos serviços de saúde, não reforçar esta atitude.

\section{Colaboradores}

D. B. Freire participou da construção do projeto, composição do banco de dados, análise e redação do artigo. L. P. Gigante auxiliou na redação do projeto, participou da coleta de dados, participou da análise e da redação do artigo. J. U. Béria coordenou a pesquisa, participou da coleta de dados, participou da análise e da redação do artigo. B. C.W. Raymann auxiliou na redação do projeto, participou da coleta de dados, participou da análise e da redação do artigo. A. C. L. Figueiredo coordenou a coleta de dados, participou da análise e da redação do artigo. L. S. Palazzo participou da redação do projeto e da análise e redação do artigo. 


\section{Referências}

1. World Health Organization. Hearing AIDS and services for developing countries. Rev Panam Salud Pública 2001; 10:139-42.

2. Torres EF, Mazzoni AA, Mello AG. Nem toda pessoa cega lê em Braille nem toda pessoa surda se comunica em língua de sinais. Educação e Pesquisa 2007; 33:369-85.

3. Ubido J, Huntington J, Warburton D. Inequalities in access to healthcare faced by women who are deaf. Health Soc Care Community 2002; 10:247-53.

4. Cardoso AHA, Rodrigues KG, Bachion MM. Perception of persons with severe or profound deafness about the communication process during health care. Rev Latinoam Enferm 2006; 14:553-60.

5. Stein A, Zelmanowicz AM, Lima AK. Promoção da saúde e detecção precoce de doenças no adulto. In: Duncan B, Schmidt MI, Giugliani ERJ, organizadores. Medicina ambulatorial: condutas de atenção primária baseadas em evidências. Porto Alegre: Artmed; 2004. p. 567-75.

6. Chaveiro N, Barbosa MA. Assistência ao surdo na área de saúde como fator de inclusão social. Rev Esc Enferm USP 2005; 39:417-22.

7. Jones EG, Renger R, Firestone R. Deaf community analysis for health education priorities. Public Health Nurs 2005; 22:27-35.

8. McAller M. Communicating effectively with deaf patients. Nurs Stand 2006; 20:51-4.

9. Béria JU, Raymann BCW, Gigante LP, Figueiredo ACL, Jotz G, Roithman R, et al. Hearing impairment and socioeconomic factors: a population-based survey of an urban locality in southern Brazil. Rev Panam Salud Pública 2007; 21:381-7.

10. Grimes DA, Schulz KF. Bias and causal associations in observational research. Lancet 2002; 359:248-52.

11. Meador HE, Zazove P. Health care interactions with deaf culture. J Am Board Fam Pract 2005, 18:218-22.

12. Steinberg AG, Wingins EA, Barmada $\mathrm{CH}$, Sullivan VJ. Deaf woman: experiences and perceptions of healthcare system access. J Womens Health 2002; 11:729-41.
13. Cooper A, Rose J, Mason O. Measuring the attitudes of human service professional toward deafness. Am Ann Deaf 2004; 148:385-8.

14. Bruin ED, Graaf R. What do we know about deaf clients after thirteen years of ambulatory mental health care? An analysis of the PsyDoN Database, 1987-1999. Am Ann Deaf 2004/2005; 149:384-93.

15. Steinberg AG, Sullivan VJ, Loew RC. Cultural and linguistic barriers to mental health service access: the deaf consumer's perspective. Am J Psychiatry 1998; 155:982-4.

16. Jones EG, Ouellete SE, Kang Y. Perceived stress among deaf adults. Am Ann Deaf 2006; 151:25-31.

17. Job J. Factors involved in the ineffective dissemination of sexuality information to individuals who are deaf or hard of hearing. Am Ann Deaf 2004; 149:264-73.

18. Joseph JM, Sawyer R, Desmond S. Sexual Knowledge, behavior, and sources of information among deaf and hard of hearing college students. Am Ann Deaf 1995; 140:338-45.

19. Luckner JL, Gonzales BR. What deaf and hard-ofhearing adolescents know and think about AIDS. Am Ann Deaf 1993; 138:338-42.

20. Brasil. Lei $n^{\circ}$. 10.098 de 19 de dezembro de 2000 . Estabelece normas gerais e critérios básicos para a promoção da acessibilidade das pessoas portadoras de deficiência ou com mobilidade reduzida, e dá outras providências. Diário Oficial da União 2000; 20 dez.

21. Brasil. Decreto no 5.296 de 2 de dezembro de 2004. Regulamenta as Leis $n^{\circ} .10 .048$, de 8 de novembro de 2000, que dá prioridade de atendimento às pessoas que especifica, e $\mathrm{n}^{\circ}$. 10.098, de 19 de dezembro de 2000, que estabelece normas gerais e critérios básicos para promoção da acessibilidade das pessoas portadoras de deficiência ou com mobilidade reduzida, e dá outras providências. Diário Oficial da União 2004; 3 dez.

22. Marín CR, Góes MCR. A experiência de pessoas surdas em esferas de atividade do cotidiano. Cad CEDES 2006; 26:231-49.

Recebido em 11/Mar/2008

Versão final reapresentada em 06/Out/2008

Aprovado em 21/Out/2008 